\title{
(Un)intended consequences of networking on individual and network-level efficiency
}

\author{
Kyosuke Tanaka* (D) and Emőke-Ágnes Horvát
}

\section{*Correspondence:}

kyosuke@u.northwestern.edu Northwestern University, School of Communication, 2240 Campus Drive, 60208 Evanston, IL, USA

\begin{abstract}
Research shows that individuals benefit from setting up their personal networks strategically. There are two fundamental networking approaches: structural change, which involves the addition and deletion of communication ties, and frequency change, which means an increase or decrease of communication on existing ties. Existing literature has focused predominantly on structural change, leaving the potential of frequency change understudied. To investigate the differential effects of structural and frequency change on the efficiency of information flow through a network, we conduct simulations of networking approaches on a diverse set of real-world directed weighted communication networks. Our results indicate that, overall, frequency change is associated with higher efficiency than structural change in all but one of the studied cases. Structural change has a strong direct effect on individuals who actively modify their personal network. Surprisingly, our simulations also show that the indirect effects of frequency change (i.e., an individual's ties are reshaped due to others' actions) can exceed direct ones. Our results highlight the value of the so far less studied frequency change and suggest that using targeted network alterations can result in gains for information flow at the scale of the entire network.
\end{abstract}

Keywords: Communication networks, Efficiency, Edge alteration, Reciprocity, Triadic closure, Computer simulations

\section{Introduction}

Individuals communicate with each other strategically to improve access to information and to capitalize on social contacts in attaining personal and professional goals. Although people hardly consider larger network structures when they communicate, to whom they choose to speak has unintended and far-reaching implications for information flow. Research in network science has studied in detail how the structure of socio-technological networks impacts human behavior (Barabási 2016; Borgatti et al. 2009; Newman 2003; Watts and Strogatz 1998). This perspective establishes that individuals' structural position predetermines their opportunities (Giddens 1984), but does not address people's ability to shape their own network to better fit individual and group needs. By contrast, recent studies acknowledge the importance of individual efforts in shaping personal networks and accessing critical information through networking (Casciaro et al. 2014; Ebbers 2014;

(c) The Author(s). 2019 Open Access This article is distributed under the terms of the Creative Commons Attribution 4.0 International License (http://creativecommons.org/licenses/by/4.0/), which permits unrestricted use, distribution, and reproduction in any medium, provided you give appropriate credit to the original author(s) and the source, provide a link to the Creative Commons license, and indicate if changes were made. 
Engel et al. 2017; Hallen and Eisenhardt 2012; Vissa 2012). This line of research defines networking as individuals' proactive and purposeful action to communicate with their social contacts (Kuwabara et al. 2018). Building on this definition, we study how various networking approaches impact the efficiency of communication networks.

We conduct data-driven computer simulations that examine the effect of two predominant networking approaches: (a) structural change, involving the addition and deletion of communication ties and (b) frequency change, involving an increase or decrease of communication on existing ties. In our proposed framework, these two approaches cover the spectrum of exploring new connections and exploiting existing ones (Aral and Van Alstyne 2011; Mason and Watts 2012; Siciliano et al. 2018; Sommerfeldt and Yang 2017; Vissa 2012). Structural change is implemented based on the generic social process of triadic closure (i.e., individuals with shared connections start to communicate with each other). The implementation of frequency change relies on interaction reciprocity (i.e., the mutuality of message exchanges between individuals). Additionally, we quantify network efficiency based on the average shortest weighted path length as a proxy for the speed at which information can be exchanged through the network (Latora and Marchiori 2001; Mason and Watts 2012). Throughout this work, we leverage real-world communication networks as the base structure for our analysis. We represent these data with weighted directed networks, to account for the fact that communication is usually initiated by one person, providing direction for interactions, and occurs repeatedly over time between interacting pairs, a detail that we model with edge weights. We analyze these networks by exploring how efficiency changes for the entire network and, more importantly, for individuals. In the latter case, we differentiate between two effects: a direct effect, impacting individuals whose neighborhood changes as a result of their own networking efforts, and an indirect effect, affecting individuals whose network is reshaped as a result of others' actions.

Our paper makes two main contributions. To further empirical inquiries in this area, the work develops a novel conceptual framework to study the effect of various networking approaches on the efficiency of communication networks. Our proposed framework indicates that triadic closure and reciprocity impact efficiency differently. Our approach enabling this finding connects existing methodologies at the level of the entire network, that is, system-level approaches from network science (Miritello et al. 2013), with the extensive study of personal or ego networks in management literature (Casciaro et al. 2014; Ebbers 2014; Engel et al. 2017; Hallen and Eisenhardt 2012; Vissa 2012). Additionally, unlike previous research focusing on forming and maintaining connections (Ebbers 2014; Engel et al. 2017; Siciliano et al. 2018; Sommerfeldt and Yang 2017; Vissa 2012), the proposed framework also covers the dissolution of social ties, which so far represent a less prominent aspect in the literature prominent aspect in the literature, with a few exceptions (Chan et al. 2014; Chen et al. 2016; Tong et al. 2012). Most importantly, the framework also includes a gradual decrease in the frequency of interactions between pairs of people. Our work thus opens new avenues to empirically test the complexity of networking.

Methodologically, we contribute to the expanding literature on edge alteration methods by proposing and implementing new approaches for weighted directed networks. Prior work on edge alteration focused solely on unweighted, undirected networks (Arrigo and Benzi 2016; Beygelzimer et al. 2005; Chan et al. 2014; Chen et al. 2016; Garimella et 
al. 2017), and as a result, has not developed edge strengthening or weakening methods. We argue that since weighted social and communication network data have increased in availability and importance (Opsahl and Panzarasa 2009), our proposed methods are not only a fundamental addition to the edge alteration literature, but also to the study of social and communication networks.

This paper extends our previously introduced framework that centered on the tensions between individual and network-level efficiency. The main idea was that when individuals optimize their personal networks, this creates inefficiencies in the network as a whole (Tanaka and Horvát 2019). In this paper, we explore the opportunities behind frequency change, especially at the individual level. The enhanced focus on the individual level is important for the entire range of cases, in which a centralized decision that shapes the whole communication system is not feasible or desired. There is also a small, but consequential, difference in one of the fundamental quantities we compute for weighted networks: our previous paper normalized the weighted shortest path length, used in the definition of efficiency, by the average path length over the entire network as suggested by Opsahl et al. (2010). Here, we use the non-normalized version of the weighted shortest path length (Newman 2001; Brandes 2001), which enables us to better study microchanges at the level of individuals. Ultimately, this is required to understand how each individual could improve his/her own networking efforts. As important steps toward this goal, we uncover both direct and indirect effects of networking in this paper. The latter account for the sometimes unintended and often hidden consequences of information flow on overall communication efficiency.

The paper is structured as follows. In "Experimental setup" section, we outline our edge alteration simulations that examine "what-if" scenarios based on different networking approaches and describe the five datasets we used throughout these simulations. Then, we detail the two main components of our framework: networking approaches and efficiency. While we use a standard way to quantify efficiency (see "Measuring efficiency" section), we propose novel models of networking approaches that alter connection structure and communication frequency (see "Modeling networking approaches" section). Our analyses explore the differences in efficiency changes across the five datasets at the system level and around individuals. We conclude with a discussion of our findings.

\section{Experimental setup} Edge alteration simulations

To examine how networking approaches change efficiency in different communication settings, we conduct data-driven computer simulations throughout which we manipulate the structure and frequency of communications based on four different networking rules (see "Modeling networking approaches" section). Our simulations are aligned with so-called edge alteration methods. Edge alteration methods are commonly used in network science and have allowed researchers to manipulate network structures to assess robustness (Beygelzimer et al. 2005; Chan et al. 2014), opinion polarization (Garimella et al. 2017) and efficiency (Arrigo and Benzi 2016; Chen et al. 2016; Tong et al. 2012). Since most research on edge alteration methods aims to optimize a certain measure, it often puts forth assumptions that overlook inherent human constraints, such as cognitive limits and attention (Arrigo and Benzi 2016; Beygelzimer et al. 2005; Chan et al. 2014; Chen et al. 2016; Garimella et al. 2017; Tong et al. 2012). For instance, (Chen et al. 2016) 
developed eigenvalue algorithms for edge addition and deletion. These algorithms choose those edges for alteration that most facilitate diffusion. Nevertheless, it is unrealistic to assume that people who are embedded in the network would have enough information to do a similar calculation and then optimize their personal connections accordingly. To address such shortcomings, the edge alteration rules that we propose are guided by social and communication theory. This increased domain-awareness can better inform personal networking in real-world settings, where direct experimentation with networking approaches could have high social costs.

We also note that edge alteration methods are different from statistical network models, such as exponential random graph modeling (ERGM) or stochastic actor-oriented modeling (SAOM). ERGM and SAOM enable researchers to estimate which micro-actions or social processes are likely to account for existing network structures (Block et al. 2016). Nonetheless, they do not necessarily explain which type of micro-action is likely to change individual and/or network-level objectives, such as the efficiency of information flow. Moreover, the current implementations of ERGM and SAOM focus more on understanding the structural features of networks than on the frequency of connections. That is, these methods reveal the presence or absence of communication ties based on specific social processes like homophily, but are oblivious to changes in the weight of edges. Thus, our edge alteration methods provide complementary insights about communication networks, in which the frequency of information exchange plays a major role.

\section{Data}

We conduct simulations based on real-world networks to obtain more realistic insights compared to synthetic toy networks. To this end, we use five communication networks. The DNC network is built from the email communication between the members of the Democratic Party around the National Committee for 17 months (Kunegis 2013). The EU-Dept4 and EU-Inst networks are based on email exchanges between affiliates of a large European research institution over one year (Leskovec and Krevl 2014). While EU-Dept4 is a sub-network that maps the communication within a department of the institution, EU-Inst is the email network of the entire institution. The MANU network comes from employee emails at a mid-size Polish manufacturer over nine months (Kunegis 2013). Finally, the OSN network maps message exchanges among U.S. college students on a social networking site for half a year (Leskovec and Krevl 2014). We obtained these datasets from different sources: The EU-Dept4, EU-Inst, and OSN networks are from Stanford Large Network Dataset Collection (Leskovec and Krevl 2014), while DNC and MANU are retrieved from the Koblenz Network Collection (Kunegis 2013). We select datasets that mainly record communication via email, as this has been the key form of computer-mediated communication for the past decades. Email usage has been found to influence productivity (Jackson et al. 2003; Mark et al. 2016), perceptions of overload (Dabbish and Kraut 2006), work-life balance (Waller and Ragsdell 2012), and trust and engagement with certain topics (Mishra et al. 2014). Most importantly, a lot of our networking happens via email, recording a near-complete trace of communication activity that we then analyze here.

Table 1 provides a summary of descriptive statistics. The five networks differ in terms of their contexts, covered time period, the number of individuals and the structural features 
Table 1 Summary statistics of the datasets: number of nodes, edges and messages; number of messages sent on average between communicating individuals (message per edge); time frame covered in the data expressed in months; reciprocity; transitivity; and efficiency $\left(E^{W}\right)$

\begin{tabular}{llllll}
\hline Data & DNC & EU-Dept4 & EU-Inst & MANU & OSN \\
\hline Nodes & 1866 & 142 & 986 & 167 & 1899 \\
Edges & 5517 & 1375 & 24,929 & 5783 & 20,296 \\
Messages & 39,264 & 48,141 & 332,334 & 82,927 & 59,835 \\
Messages per edge & 7.117 & 35.012 & 13.331 & 14.340 & 2.948 \\
Time frame (in months) & 17 & 18 & 18 & 9 & 6 \\
Reciprocity & 0.411 & 0.788 & 0.711 & 0.876 & 0.636 \\
Transitivity & 0.089 & 0.735 & 0.267 & 0.541 & 0.057 \\
EW $^{W}$ & 0.385 & 5.279 & 6.909 & 15.817 & 0.811 \\
\hline
\end{tabular}

of the emerging communication networks. For instance, the smallest network (EU-Dept4) is one order of magnitude smaller than the largest one (OSN), and the most densely connected network (MANU) is considerably denser than the least connected network (DNC). More importantly, the efficiency (see Eq. 1 in "Measuring efficiency" section) ranges between 0.385 and 15.817, thus spanning two orders of magnitude.

To consistently model networks of different digital communication traces, we construct weighted directed networks that capture how frequently a specific individual messaged another person. In network terms, we build cross-sectional weighted edge lists from temporal data and then perform the simulations outlined below.

\section{Modeling networking approaches}

We define networking as individuals' proactive and purposeful action to communicate with their social contacts (Kuwabara et al. 2018). According to this definition, networking is an action taken by the individual. As prior research asserted, those who network well accrue benefits, such as better access to information and more career success (Vissa 2012; Wolff and Moser 2009). Nonetheless, networking also impacts those neighbors whose network is reshaped as a result of another person's networking. In this paper, we demonstrate both direct and indirect effects of networking on information flow, and contribute to the argument that it is important to understand both effects (Miritello et al. 2013). To complement existing qualitative research centered on personal networking (Ebbers 2014; Engel et al. 2017), we put our individual-level findings into a system-wide perspective as well, showing the benefits and drawbacks of networking, beyond one's immediate neighborhood.

Prior literature has identified two main approaches to networking (Aral and Van Alstyne 2011; Siciliano et al. 2018; Sommerfeldt and Yang 2017; Vissa 2012). As summarized in Fig. 1, the first approach centers on structural change by expanding or narrowing the set of existing communication partners, while the second approach focuses on frequency change, by increasing or decreasing the prevalence of interactions between connected pairs. While structural change enables individuals to connect with a diverse set of contacts, frequency change is related to creating networks of strongly connected individuals (Aral and Van Alstyne 2011). These approaches map intuitively to the principles of exploration (i.e., choosing an unfamiliar option with unknown outcomes) and exploitation (i.e., choosing a familiar option with known outcomes) found in learning problems across human decision-making and computer science (Aral and Van Alstyne 2011; Auer et 


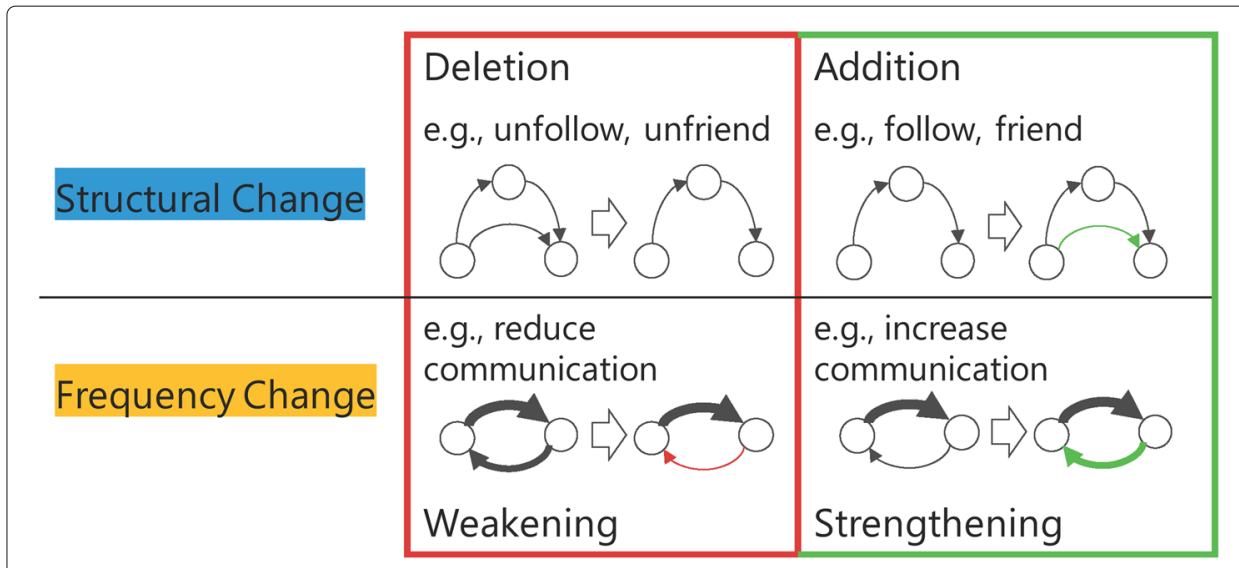

Fig. 1 Considered networking approaches

al. 2002; He and Wong 2004; Lazer and Friedman 2007; Mason and Watts 2012; Siciliano et al. 2018).

Building on the trade-off between exploration and exploitation, one can argue that structural change is less likely to occur because it is more costly than frequency change. First, structural change requires searching and identifying new contacts (Siciliano et al. 2018; Vissa 2012). This exploration is costly: since information about new contacts is limited, it is uncertain whether it is worth investing in a potential new contact. Exploration is a process in which individuals expand their contacts (variation) and select some contacts over others (selection), as argued by March (1991). In this sense, stopping interaction with an existing contact, which is another way to alter network structure, can also be considered a potentially expensive form of exploration (Siciliano et al. 2018).

Additionally, frequency change might be more straightforward and less costly than structural change because it is mainly a form of exploitation where individuals repeat their existing communication patterns. Changing the frequency of interactions with established contacts is less risky (Vissa 2012). These arguments point to the importance of understanding the so far understudied frequency change.

Certainly, most individuals engage in a sustained combination of exploration and exploitation, regularly altering both their connections and the prevalence of their interactions. We thus study both structural and frequency change and compare how both affect not only those individuals whose neighborhood changes as a result of their own networking efforts, but also those whose network is reshaped as a result of others' actions.

\section{Structural change}

Structural change in the form of adding new and deleting existing communication ties is common in every communication context (Siciliano et al. 2018). Thus, most literature on networking focuses on this form of networking. For instance, several studies have been dedicated to understanding how individuals form new connections with potential partners in business contexts (Ebbers 2014; Engel et al. 2017; Hallen and Eisenhardt 2012). Equally often, people stop communicating with their contacts. This process is apparent on social media to the extent where Facebook coined the term unfriend (Peña and Brody 2014) and Twitter introduced the usage of the new verb unfollow (Kwak et al. 2011; Xu 
et al. 2013). Since in any communication setting there are several possibilities to decide which ties to create and/or remove, we use key observations from social network analysis to model the addition and deletion of connections. These models are informed by key social processes and distinguish our research from previous work on edge addition and deletion (e.g., Chen et al. 2016; Tong et al.2012).

\section{Adding Edges}

Expanding one's existing contacts plays a key role in accessing new information. Hence, several of our networking attempts are aimed at creating new contacts (Vissa 2012). As suggested by the pervasiveness of connecting to friends of friends, the fundamental tendency of triadic closure indicates that ties are most likely to form naturally between individuals who have a mutual friend (Estrada and Arrigo 2015; Faust 2010). This observation will guide our models of edge addition as we create a new communication tie between two people with a shared contact who have not been previously communicating with each other.

For the implementation of our addition rule, we define the neighbors of node $A$ as those individuals who have either sent message(s) to, received message(s) from, or have been mutually communicating with $A$. Then, we (1) pick a random person with at least two unconnected neighbors from the network, (2) randomly select two of their unconnected neighbors, and (3) connect these neighbors with an edge weight of 1 , randomly determining the directionality of the new edge. We repeat these steps until the desired number of edges are added.

\section{Deleting Edges}

By reducing the number of communication ties, people can cultivate their existing relationships. In network terms, this maps to a process of edge deletion that is typically associated with amplifying the bandwidth of the remaining communication ties. Existing triangles ( $A$ connected to $B$ connected to $C$ connected to $A$ ) often break for two reasons: to avoid conflict, as predicted by structural balance theory (Faust 2010), and to channel communication through the shared contact who gains information advantages from this bridging position (Burt 1992). Our edge deletion heuristic will thus enable us to study how increasing the number of unclosed triangles in a communication network changes network-level and individual information flows.

Accordingly, our deletion rule (1) picks a random person with at least two connected neighbors, (2) randomly selects two of the connected neighbors, and (3) eliminates a connection between the neighbors. As in the case of the edge addition, these steps are repeated until the specified number of edges get removed. The addition and deletion of edges as approaches to change the structure of communication networks are summarized in the top panel of Fig. 1.

\section{Frequency change}

The key novelty of our paper relies on the proposed models for frequency change that focus on how individuals allocate their time and attention to existing contacts. Humans are, on average, limited to 150 relationships at a specific time point, as argued based on the social brain hypothesis (Dunbar 1998) and according to findings of limited attention and energy (Aral and Van Alstyne 2011). More importantly, the attention given to individual 
contacts varies strongly: using large-scale mobile communication data, Miritello et al. (2013) found that communication capacity at a certain time is fairly constant across individuals, yet there are significant differences in terms of whom they frequently call among their contacts. These arguments support the observation that people tend to maintain a certain number of active communication partners. As in the case of structural change, the range of possible networking approaches is extensive. In what follows, we will distill two simple heuristics to model increasing and decreasing communication frequencies on existing ties.

\section{Strengthening edges}

Reciprocation or withholding interaction is a fundamental mechanism throughout the process of differentially allocating time and energy to certain communication partners (Block 2015; Squartini et al. 2013). Reciprocity is broadly defined as an "exchange of any resource to equalize the relationship" (Casciaro et al. 2014, p.709). It helps build and access social capital by increasing communication frequency between a pair of people to balance their interactions (Siciliano et al. 2018; Sommerfeldt and Yang 2017). Reciprocity has also been shown to have become an essential norm in digital communication (Akoglu et al. 2012). For these reasons, we base our approach to strengthening ties on reciprocity. Implicitly, enhancing reciprocity will increase communication frequency.

Our implementation of the strengthening rule increases the weight of the existing communication tie from $A$ to $B$ if $B$ frequently messaged $A$ previously, but has not reciprocated these messages. In every step of the process, we focus on the most imbalanced connection between $A$ and its neighbors. Specifically, we (1) pick a random person from the network, (2) select their neighbor with whom they have a maximally imbalanced tie, and (3) increase by 1 the weight of the connection between the focal person and their neighbor. We repeat this until a given number of connections have been strengthened across the network.

\section{Weakening Edges}

Instead of suddenly dropping a contact, people typically progressively dissolve certain relationships. When it comes to communication, most of us gradually reduce contact with a partner (Raeder et al. 2011). For instance, using longitudinal data on professional connections between investment bankers, Burt (2002) found that their interactions often decayed over time. More recently, studies have investigated how people's friendships and mobile communication deteriorate (Kleinbaum 2018; Miritello et al. 2013). Based on this research, weakening ties is thus ubiquitous in the course of all sorts of social interactions.

To model how individuals re-allocate time spent on emailing certain partners, we weaken existing connections by gradually discouraging the reciprocation of interactions. Our implementation targets the most balanced conn ections as follows: (1) pick a random person, (2) select a neighbor such that the email exchange rate between the two is maximally similar, and (3) decrease by 1 the higher weight connection between the person and this neighbor, which is the equivalent of dropping one message. We repeat these steps until connection weights have been weakened a predefined number of times.

The bottom panel of Fig. 1 summarizes frequency change in the form of strengthening and weakening existing connections. Before we use this framework to examine the effect 
of networking approaches on the efficiency of communication networks, we discuss the used measure of efficiency.

\section{Measuring efficiency}

A useful notion of efficiency in our context is described by the speed at which information can spread through a network (Mason and Watts 2012). We thus evaluate the efficiency of a communication network by its capability to enable information exchange between individuals promptly (Boccaletti et al. 2006; Latora and Marchiori 2001; 2003). The most common approaches approximate the ease of transmission across a network by the shortest paths between pairs of people. Following Latora and Marchiori (2001), we use the average distance across all pairs of nodes in the quantification of the efficiency of a communication network. Note, however, that other measures have also been proposed. Most relevantly, the so-called communicability, which is built around the idea that in many real-world situations, the communication between pairs of nodes does not only take place on the shortest path between them. Instead of focusing on the shortest path, the communicability concept is centered on the total number of different paths that exist between two individuals (Estrada and Hatano 2008; Estrada et al. 2012). While this is a sensible argument, the measure effectively introduces the alternative assumption that the more paths between two nodes, the faster the message transmission. Additionally, given the intricate connection structure of large-scale networks, communicability takes very large values that make its interpretation hard. For this practical reason, we adopt the more common approach and use the widespread measure described next in the case of weighted networks.

In unweighted networks, the shortest path $d_{i j}$ between two specific individuals $i$ and $j$ is defined as the path having the minimum number of edges. In weighted networks, multiple interactions between the same pair of individuals $i$ and $j$ are coded as weights $w_{i j}$, while the length of the edge is defined as the inverse of the weight $l_{i j}=\frac{1}{w_{i j}}$ and can be used to indicate how "far" $i$ and $j$ are. The weighted shortest path $d_{i j}^{w}$ is then defined as the one with the smallest sum of edge lengths $l_{i j}$ across all possible paths in the network running from $i$ to $j$. Note that in a weighted network, a path with the minimum number of edges is not necessarily the shortest weighted path. Figure 2 illustrates the difference between the unweighted and weighted shortest path length: the shortest path between nodes $A$ and $D$ is 2 (see green path in Fig. 2b). However, if the edge between $B$ and $D$ has weight 10 (i.e., length 1/10), while the edges from $B$ to $C$ and $C$ to $D$ have weights 20 and 50,

a

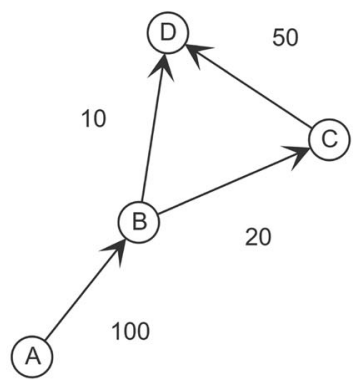

b

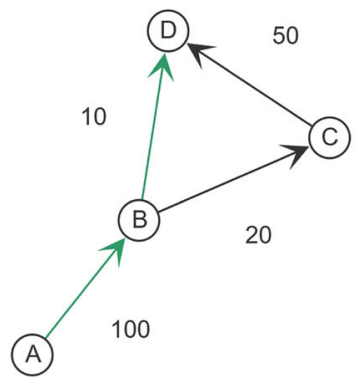

C

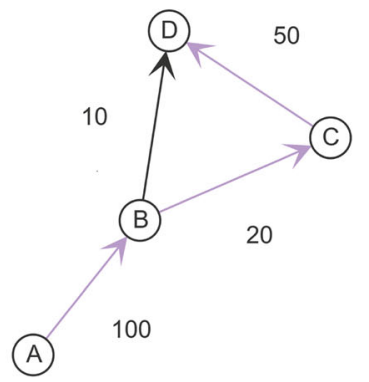

Fig. 2 Unweighted vs. weighted shortest path length. a A toy network. b Unweighted shortest path between $A$ and $D$ in green. c Weighted shortest path between $A$ and $D$ in purple 
respectively, then the weighted shortest path between $A$ and $D$ goes through $B$ and $C$ and has a total length of $1 / 100+1 / 20+1 / 50$ as opposed to $1 / 100+1 / 10$ (see purple path in Fig. 2c). The weighted shortest path is identified by inverting the edge weights and then using Dijkstra's algorithm (Brandes 2001; Dijkstra 1959; Newman 2001).

To characterize information exchange at the network level, we compute the typical separation between any pair of individuals based on the following average efficiency:

$$
E^{w}=\frac{1}{N(N-1)} \sum_{i \neq j} \frac{1}{d_{i j}^{w}}
$$

where $N$ is the number of individuals in the network and the summation goes over all pairs of individuals. Note that through the use of the inverted distance, we avoid divergence problems when the network has disconnected components, and thus the distance between nodes is formally equal to infinity. This measure has been widely applied across different domains, ranging from social to technological (Buhl et al. 2004; Latora and Marchiori 2005; Migliano et al. 2017).

\section{Results}

In this section, we report findings organized into two parts. The first part shows how different networking approaches affect efficiency across the five considered datasets. The second part describes how networking approaches impact individuals directly or indirectly, illustrating the findings on two datasets.

\section{Networking approaches and overall changes in efficiency}

Figure 3 shows the efficiency under different networking approaches. We alter the network by modifying up to $10 \%$ of the observed edges. On the right side of the plot, we add/strengthen $1 \%, 2 \%, \ldots 10 \%$ of the edges, while on the left side, we delete/weaken $1 \%$, $2 \%, \ldots 10 \%$. Each data point averages over 100 simulations and shows the corresponding standard deviation. In the DNC network, adding connections (blue, between $0 \%$ and $10 \%$ ) increases efficiency at a lower rate than strengthening connections. Deleting connections (blue, $-10 \%-0 \%$ ) has a larger impact on efficiency than weakening them. Strengthening connections (yellow, 0\%-10\%) achieves the highest increase in efficiency. By contrast, weakening connections (yellow, $-10 \%-0$ ) has a relatively small impact on efficiency. Thus, in the DNC network, efficiency decreases in the presence of edge deletion and increases most through strengthening connections.

Across all studied networks, the improvement in efficiency is most prominent when strengthening connections. Edge addition increases efficiency only slightly. Weakening edges has a negative impact on efficiency in every case, while deleted edges yield the biggest drop in all networks but OSN. Thus, our results show that structural and frequency change have asymmetric effects: whereas edge strengthening has a stronger effect on efficiency than edge addition, edge weakening is likely to have a weaker effect than edge deletion. Accordingly, frequency change is associated with higher efficiency than structural change in nearly all of the studied cases.

\section{Networking approaches and changes in individual efficiency}

To better understand the emerging differences between structural and frequency change, we investigate whether a change in outdegree (i.e., the number of people a focal individual 


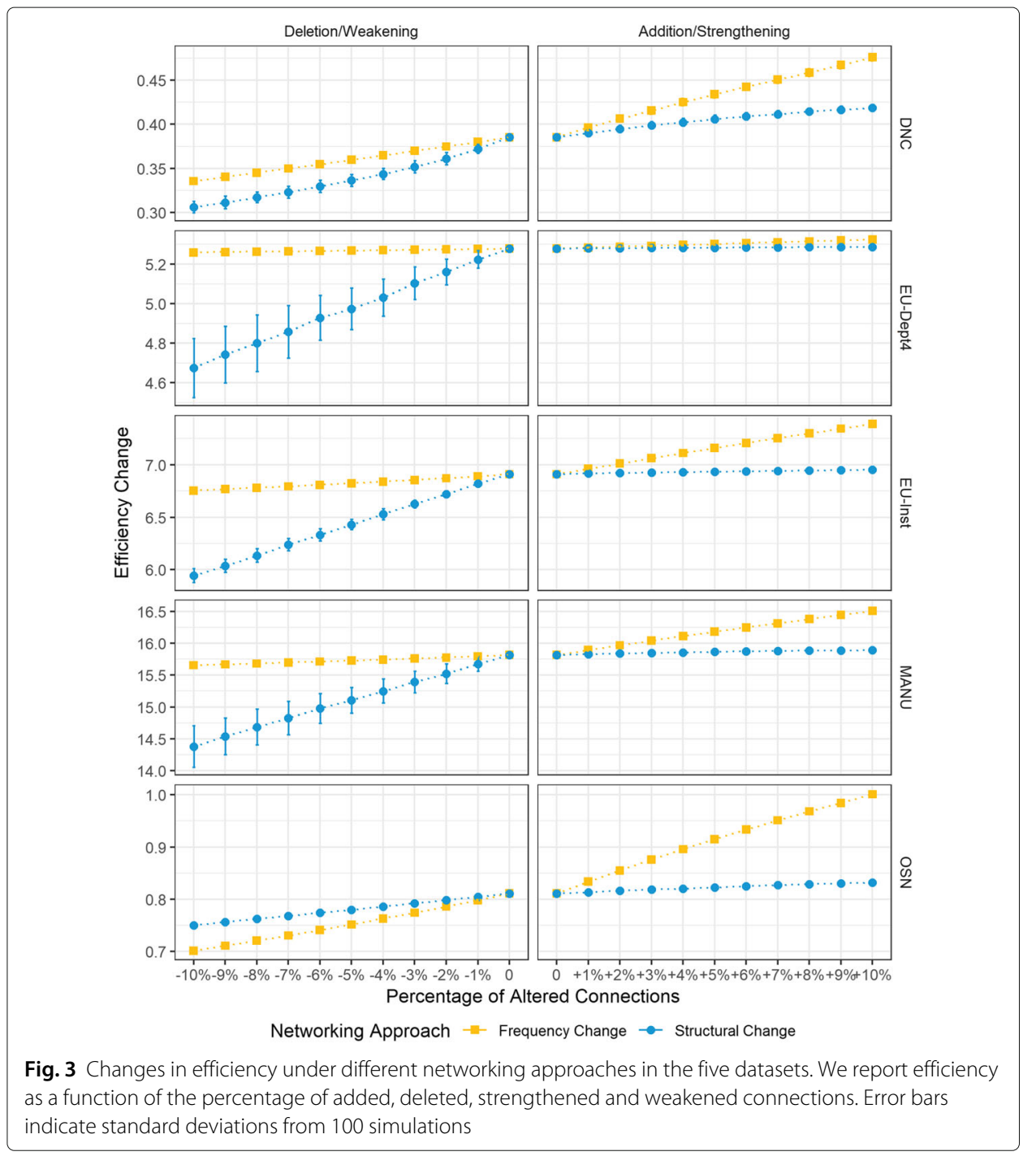

emails) is also associated with changes in efficiency. These analyses are conducted at the individual level, meaning that they establish the link between each individual's efficiency and outdegree. First, we take the average efficiency over 100 simulations for each person in the five datasets under each of the conditions (i.e, added/strengthened and deleted/weaken connections). Then, we calculate the difference between the original value and $\pm 10 \%$ of altered connections. This enables us to investigate how individuals are affected by different networking approaches. We illustrate the results on the EU-Inst and OSN networks and show similar plots for the remaining three networks in the Appendix.

Figure 4a shows the results for EU-Inst. In the case of $10 \%$ strengthened connections, there is hardly any change in outdegree (top panel, green). As expected, edge addition is associated with change in degree (bottom panel, green). It is interesting, however, that the few individuals who experience an increase in efficiency only change their outdegree by 1 at most. We find a similar separation into two nearly disjointed categories in the case of $10 \%$ weakened connections (top panel, red): almost exclusively, people either lose connections (i.e., change their outdegree) without significant associated changes in efficiency, or they experience a decrease in efficiency without a drop in their outdegree. 


\section{a}

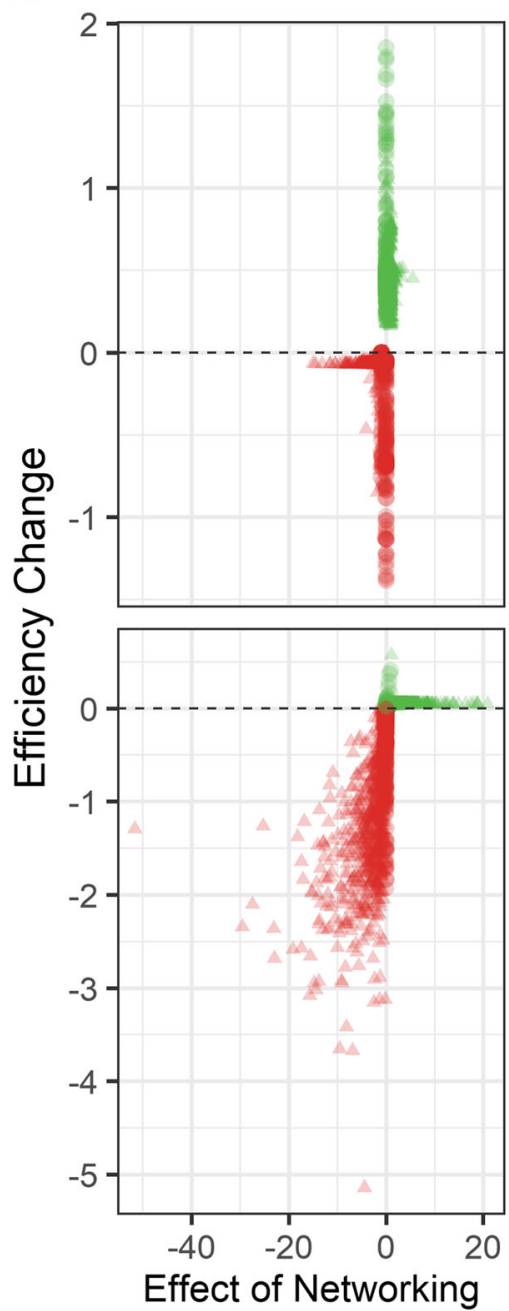

b
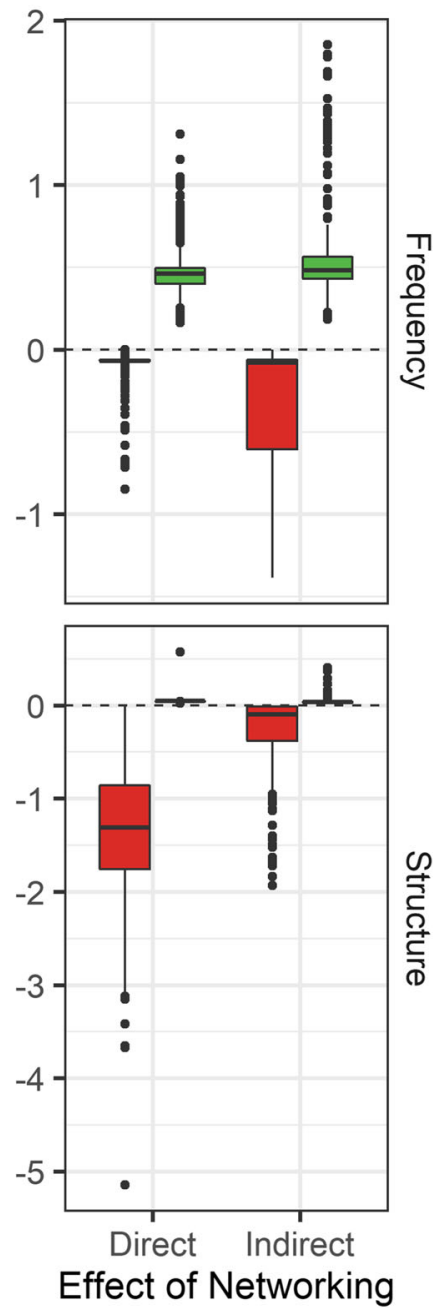

Adding/Strengthening

Fig. 4 a Changes in individual's efficiency vs. changes in their outdegree under different networking approahces based on the EU-Inst dataset. Efficiency is reported as the difference between the original data and $10 \%$ of deleted/weakened connections (red) as well as 10\% added/strengthened connections (green). b Changes in individual's efficiency in case of direct and indirect networking effects

Finally, when edges are deleted (bottom panel, red), the change in outdegree is correlated with the change in efficiency. To quantify the correlation between the changes in outdegree and efficiency, we use Kendall's $\tau$. This shows a positive correlation in the case of edge addition ( $\tau=0.491, p<0.001$ ) and deletion $(\tau=0.564, p<0.001)$. Contrarily, changes in outdegree are negatively correlated with changes in efficiency in the presence of strengthening ( $\tau=-0.059, p<0.05)$ and weakening $(\tau=-0.065, p<0.01)$.

The separation into two scenarios seen in Fig. 4 a suggests that there are two different types of effects experienced, on average, by individuals in this communication network. Our intuition is that individuals will see different changes in efficiency based on differences in their agency. In other words, we identify individuals who "actively" change their outdegree and outflow (i.e., their communication frequency with existing 
contacts) as a result of being selected throughout our simulations because they were adjacent to a chosen edge. We call this the direct effect and the formal condition for it is: $\delta_{\text {outdeg }} \geq 1 \wedge \delta_{\text {outflow }} \geq 1$. When this condition does not hold, we establish an indirect effect. This impacts individuals whose network neighborhood changes as a result of others' networking, without them taking explicit networking actions.

Figure $4 \mathrm{~b}$ compares the change in efficiency for individuals grouped into the direct and indirect effect conditions. First, this segmentation of the data allows us to quantify the extent to which efficiency is modified despite the fact that some individuals did not modify the structure or frequency of their connections as part of our algorithms. In the case of frequency change, the median indirect effect on efficiency of strengthening and weakening is $0.481(n=206)$ and $-0.078,(n=310)$, respectively. Remarkably, the effect size in case of indirect strengthening is slightly larger as the corresponding direct effect $(M d n=0.459$, $n=780$ ). Moreover, the indirect weakening effect is considerably larger than the direct weakening effect $(M d n=-0.068, n=676)$. A Wilcoxon rank sum test shows that these differences between direct and indirect effects are significant in both cases (strengthening: $W=61,632, p<0.001$; weakening: $W=132,420, p<0.001$ ). This supports the finding that in the case of EU-Inst, the indirect effect of frequency change is stronger than the direct effect.

By contrast, in the presence of structural change, indirect effects are weaker than direct effects (addition: $W=175,180, p<0.001$; deletion: $W=18,862, p<0.001$ ). The individual median effects are as follows: the direct effect of deletion $(M d n=-1.311, n=$ 627), the direct effect of addition $(M d n=0.043, n=693)$, the indirect effect of deletion $(M d n=-0.099, n=359)$, and the indirect effect of addition $(M d n=0.035, n=293)$. Notice here that direct deletion has the strongest effect on efficiency.

Next, we examine similar results for the OSN network because this data showed different trends in the first part of our analysis (see Fig. 3). The relationship between changes in individuals' efficiency and their outdegree based on different networking approaches qualitatively yields similar trends in all aspects to EU-Inst. Namely, while the correlation in case of frequency is negative (strengthening: $\tau=-0.183, p<0.001$; weakening: $\tau=-0.298, p<0.001$ ), it becomes positive in the presence of structural change (addition: $\tau=0.553, p<.0001$; deletion: $\tau=0.580, p<0.001$ ). Furthermore, Fig. 5 a reiterates that with frequency change, in particular, weakening of edges is associated with stronger indirect effects $(M d n=-0.086, n=1160)$ compared to direct effects $(M d n=-0.053, n=739)$. This difference is statistically significant ( $W=590,230, p<0.001$ ). There is no statistically significant difference between the direct and indirect effects of strengthening ( $W=$ $470,820, p=0.082$ ). By contrast, structural change has a stronger direct effect (addition: $M d n=0.023, n=829$; deletion: $M d n=-0.102, n=604$ ) than indirect effect (addition: $M d n$ $=0.016, n=1070$; deletion: $M d n=-0.022, n=1295$ ). The stronger direct effect is also supported by a Wilcoxon rank sum test for addition ( $W=634,620, p<0.001)$ and deletion ( $W=65,258, p<0.001$ ). Similar trends are also observed in Fig. 5b, where we calculate the difference between the original value and $\pm 5 \%$ of altered connections. This suggests that the shown trends are not singular to the chosen edge alteration threshold.

Finally, we investigate the main difference between the results of EU-Inst and OSN: the effect size of edge deletion. Changes in efficiency in the presence of deletion are an order of magnitude larger in EU-Inst than in OSN. In Eu-Inst, the biggest change affected not the individual with the highest number of contacts (i.e., 167), but the person who 


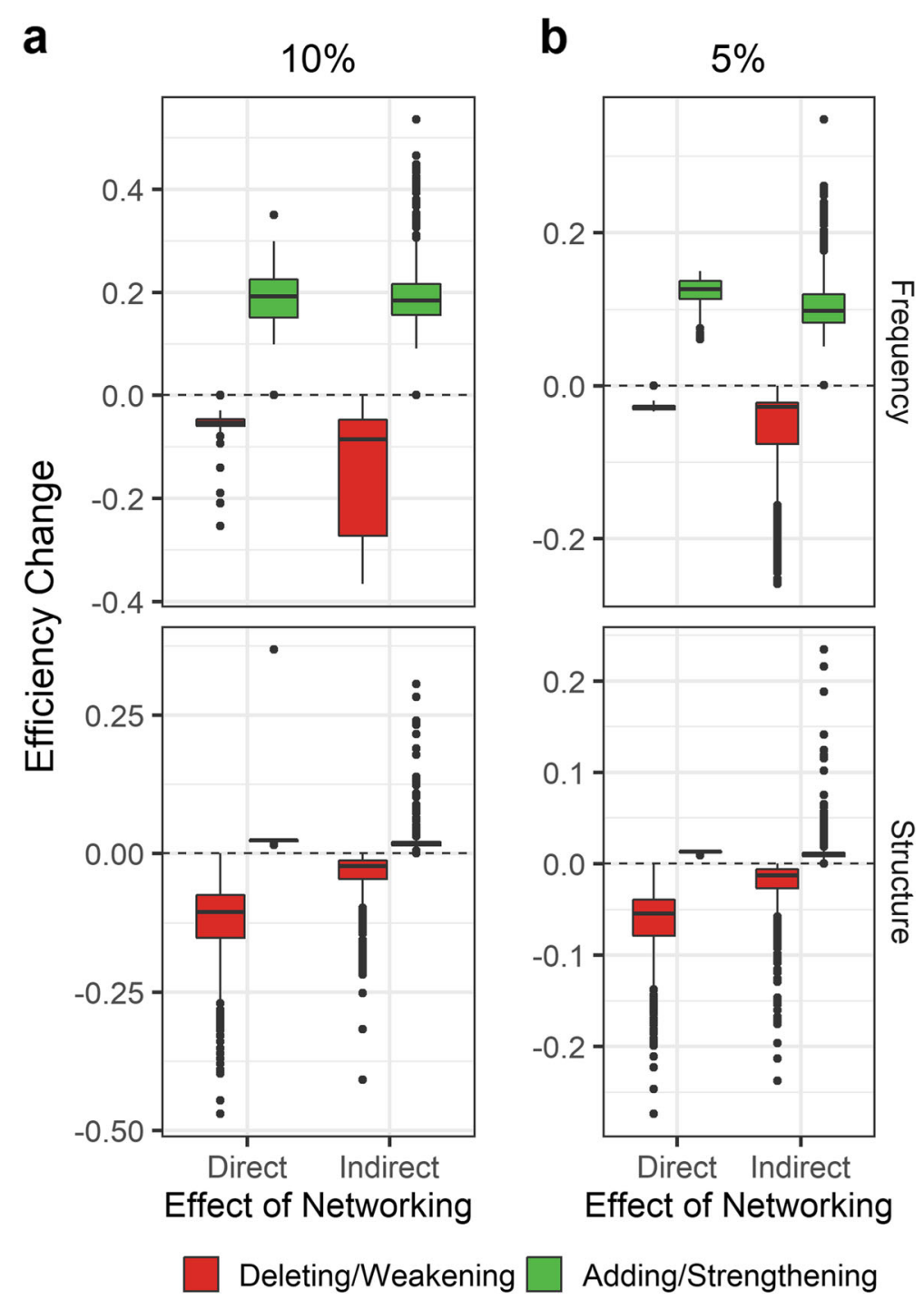

Fig. 5 a Changes in individual's efficiency in case of direct and indirect networking effects based on the difference between the original data and $10 \%$ of deleted/weakened connections (red) as well as 10\% added/strengthened connections (green) in the OSN dataset. $\mathbf{b}$ The same quantities in the same dataset based on the difference between the original data and $5 \%$ of deleted/weakened/added/strengthened connections

most frequently communicated with their 64 contacts (i.e., 4607). By contrast, in OSN, the largest change in individual efficiency happened neither in the case of the person with the highest number of contacts (i.e., 237) nor the person with the highest frequency of communication (i.e., 1091). Instead, it affected an individual with an average outdegree (i.e., 26) and outflow (i.e., 26). These exploratory observations indicate that extending our descriptive analyses with predictive components will represent non-trivial challenges. In addition, there are topological differences between EU-Inst and OSN (see Table 1). We assume that the discrepancies in messages per edge are likely to impact the effect size of edge deletion and edge weakening. OSN's messages per edge (i.e., the average edge weight) are lowest at 2.948, and the same quantity for EU-Inst is 13.331. This means that in order to actually delete an edge in OSN, we need to weaken it three times. In EU-Inst, it is considerably harder to attain the deletion effect through edge weakening since this requires, on average, 13 weakening steps. 


\section{Discussion}

We have investigated how basic networking approaches impact the efficiency of communication networks overall and at the level of individuals. Our results highlight the importance of combining computer simulations with real-world data to understand changes in paths for information flow. Based on the results of our data-driven computer simulations, at a network level, frequency change tends to be associated with higher efficiency than structural change, establishing the importance of increasing or decreasing communication frequency with current partners, as opposed to establishing new or dissolving existing connections. Efficiency can thus be grown through increasing communication frequency via enhanced reciprocity of interactions. These findings highlight that the so far less studied frequency change produces valuable results for network engineering that are worthy of further investigation. In obtaining these results, we measured efficiency using weighted path lengths. Unlike unweighted shortest paths, weighted shortest paths are not always the shortest in terms of the number of edges (cf. Fig. 2). Our findings suggest that reciprocity of interactions and triadic closure play different roles in weighted directed networks compared to their effects in unweighted undirected networks. As Opsahl et al. (2010) argued in a broader methodological context, this is an important modeling detail, especially for the study of social and communication networks. Our results benefited from the inclusion of this more involved modeling framework.

As further innovations to the framework, we not only applied existing edge alteration methods to study the effect of networking, but also implemented a novel approach consisting of frequency change. Most work on edge alteration has been based on addition and deletion in unweighted networks (Arrigo and Benzi 2016; Chan et al. 2014; Chen et al. 2016; Garimella et al. 2017; Tong et al. 2012), but this paper explored how altering communication frequency can have as large an effect on efficiency as structural change (addition or deletion of connections), which can be an important consideration in ongoing edge alteration research. Given that communication should focus not only on whom you communicate with but also on how often you communicate (Aral and Van Alstyne 2011; Monge and Contractor 2003), our method contributes to the investigation of communication networks in a novel way.

At the level of individuals, we found that they are indirectly affected by changes in their neighborhoods and that these indirect effects are substantial, especially in the case of frequency change. This finding confirms that the frequency of communication between a pair of individuals is essential to obtain novel information, as suggested by the diversity bandwidth trade-off theory (Aral and Van Alstyne 2011). This theory asserts that there is a compromise between having a diverse set of contacts vs. the frequency of interactions, with high-frequency connections (i.e., strong ties) playing a key role in accessing novel information. Our results also highlight the importance of having high-frequency connections to improve efficiency. Since the diversity bandwidth trade-off theory focuses on similar personal networks as the ones studied here, our findings contribute to current knowledge by also taking important indirect effects into account.

Finally, we contribute to the literature on networking by demonstrating direct vs. indirect effects of networking on the efficiency of information flow. Previous studies mainly focused on understanding the intent of networking and its consequences (Ebbers 2014; Engel et al. 2017; Siciliano et al. 2018; Sommerfeldt and Yang 2017; Vissa 2012) and principally overlooked unintended consequences of networking. Our results show that as an 
effect of networking, some alters can benefit from others' networking action. In particular, our simulation results indicate that frequency change has stronger indirect effects than structural change. This externality should be incorporated into networking theories in the future and should also be tested based on further empirical data in various contexts. Indirect effects of networking, we find, considerably enrich our understanding of networking.

\section{Limitations and future directions}

We address here the limitations of our work that provide promising opportunities for future research. First, we focused solely on modeling two out of several possible networking approaches. Prior research has begun to explore alternative algorithms that model networking (Arrigo and Benzi 2016; Chan et al. 2014; Chen et al. 2016; Garimella et al. 2017; Tong et al. 2012). However, these studies have not yet examined weighted networks and frequency changes. Given our results, it is important for future work to investigate how individual and system-level characteristics change in accordance with different networking approaches.

Second, we did not investigate the content of digital communication. Although this was beyond the scope of our study, we acknowledge the importance of examining how simple vs. complex knowledge is transmitted and which networking approaches work best in different cases. Accordingly, future research could investigate the content and context of messages in addition to networking approaches, as the linguistic markers of conversation will have major consequences for the success of networking. We expect such research to expand the measure of efficiency by taking into account the heterogeneity of the communication that circulates through email (from the mundane to the critical). For example, one could re-weight email exchanges based on different notions of importance and define efficiency in terms of the spreading speed and reach of "important" information.

Third, although the used network efficiency measure has been widely adopted in network science (Boccaletti et al. 2006; Latora and Marchiori 2001; 2003), our findings might not be generalized to other types of outcome measures, such as betweenness centrality (Freeman 1977; Brandes 2001) and communicability (Estrada and Hatano 2008; Estrada and Arrigo 2015). Given that the goals of networking often differ by individuals in organizations, future research should study how different networking approaches affect other outcome measures.

Finally, there is great potential in extending the presented framework. It would be useful to add external factors to the model, such as node attributes and different types of social relations. Furthermore, instead of implementing the model with random node selection, one can extend the model by devising targeted selection approaches such as the preferential selection of nodes based on their degree (Watts 2004 pp. 279-285). Also, while we manipulate networks using one approach at a time, one might combine different networking approaches to simulate other potential consequences.

\section{Conclusion}

In this paper, we reported results on the effects of networking in social and communication networks modeled by directed weighted networks. Interestingly, the analysis of our data-driven simulations suggests that changes in the frequency of interactions 
significantly improve or deteriorate efficiency both at a network and individual level. Additionally, our simulations demonstrate an indirect effect of the two studied networking approaches on information flow, highlighting that the thus far less studied frequency change produces valuable outcomes for network engineering. Therefore, we believe that further investigations in this area can provide a fruitful direction in social and communication network research.

\section{Appendix}

Here we provide figures showing changes in individuals' efficiency in the networks DNC (see Fig. 6), EU-Dept4 (see Fig. 7), and MANU (see Fig. 8).

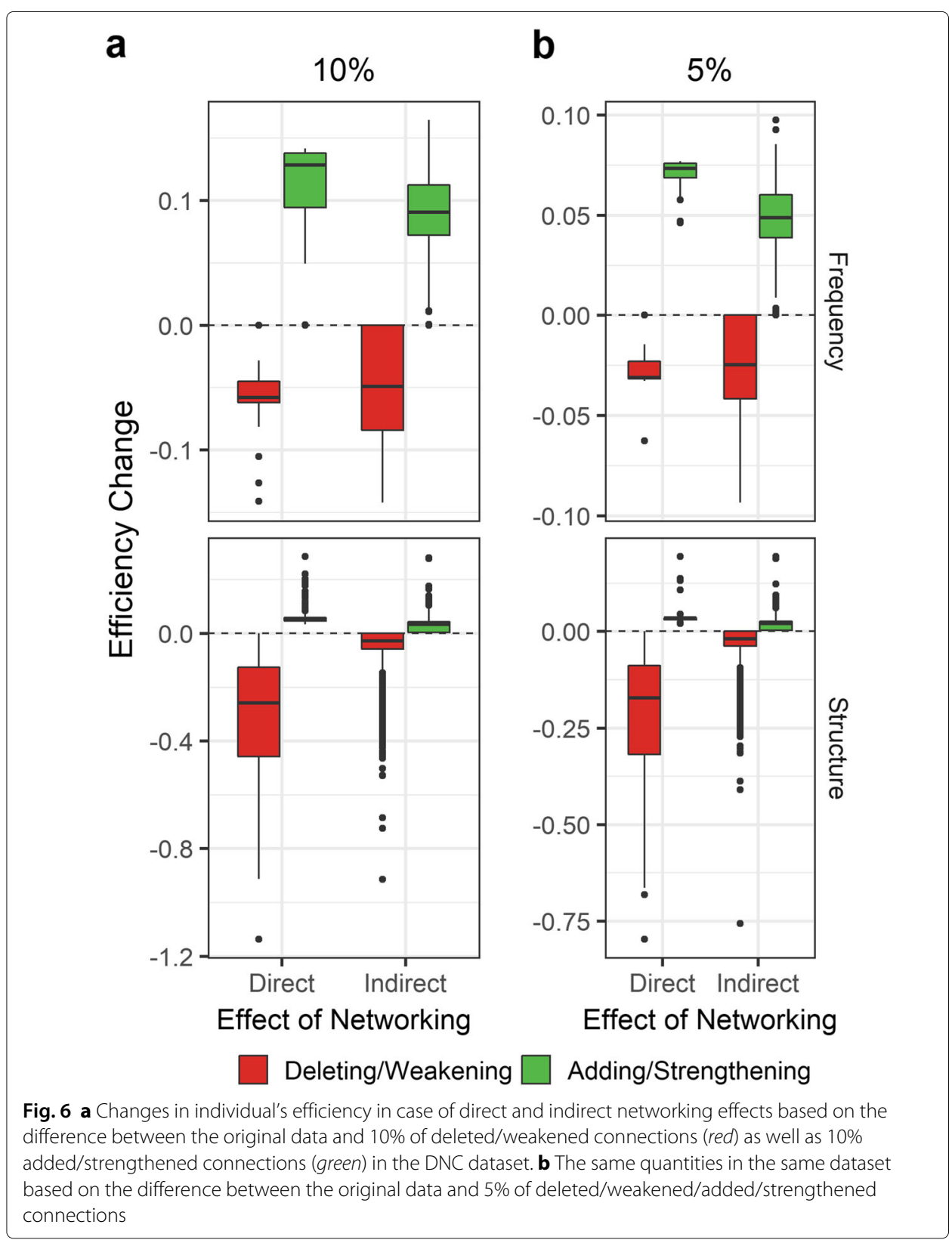



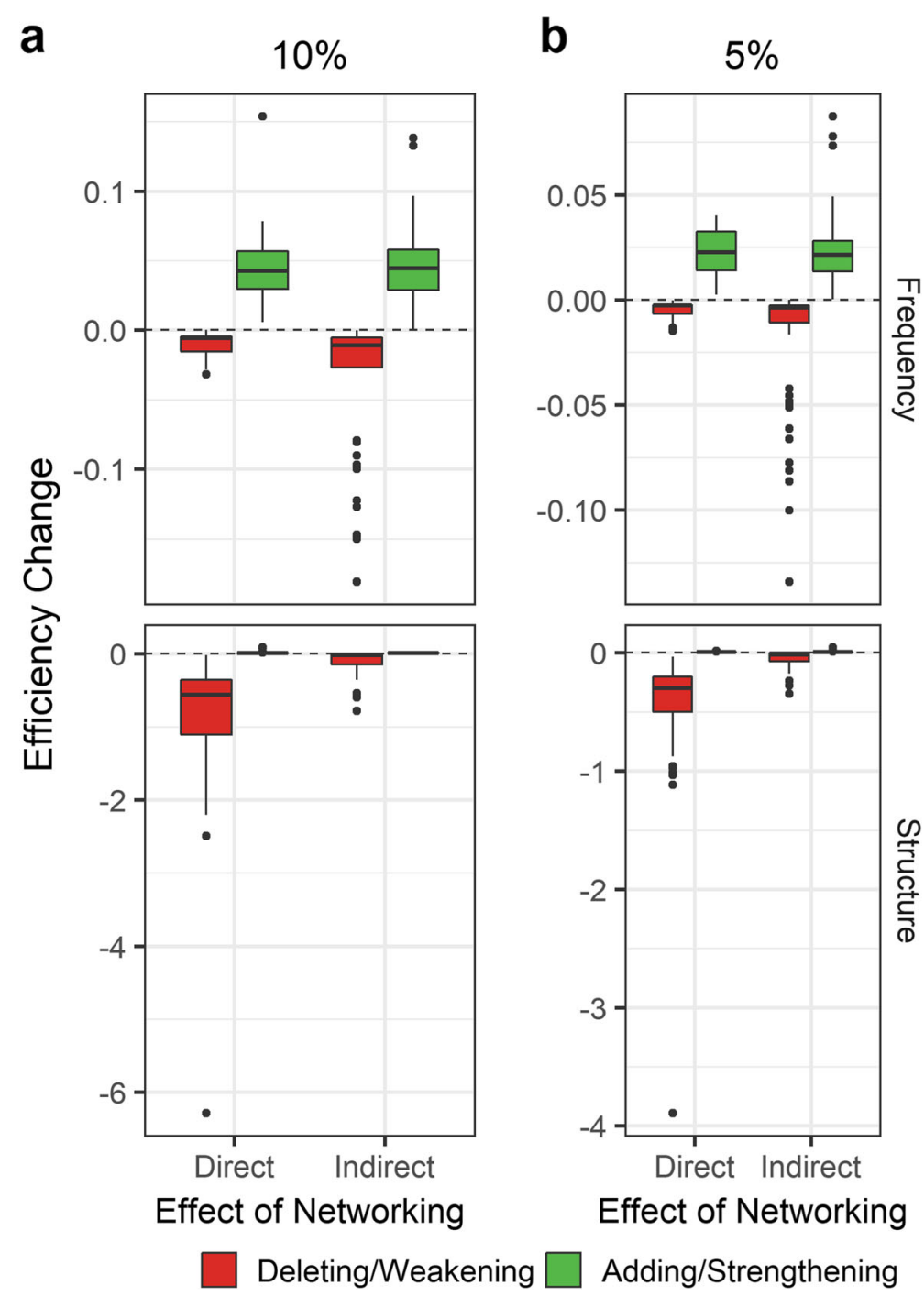

Effect of Networking

\section{Adding/Strengthening}

Fig. 7 a Changes in individual's efficiency in case of direct and indirect networking effects based on the difference between the original data and 10\% of deleted/weakened connections (red) as well as 10\% added/strengthened connections (green) in the EU-Dept4 dataset. b The same quantities in the same dataset based on the difference between the original data and $5 \%$ of deleted/weakened/added/strengthened connections 


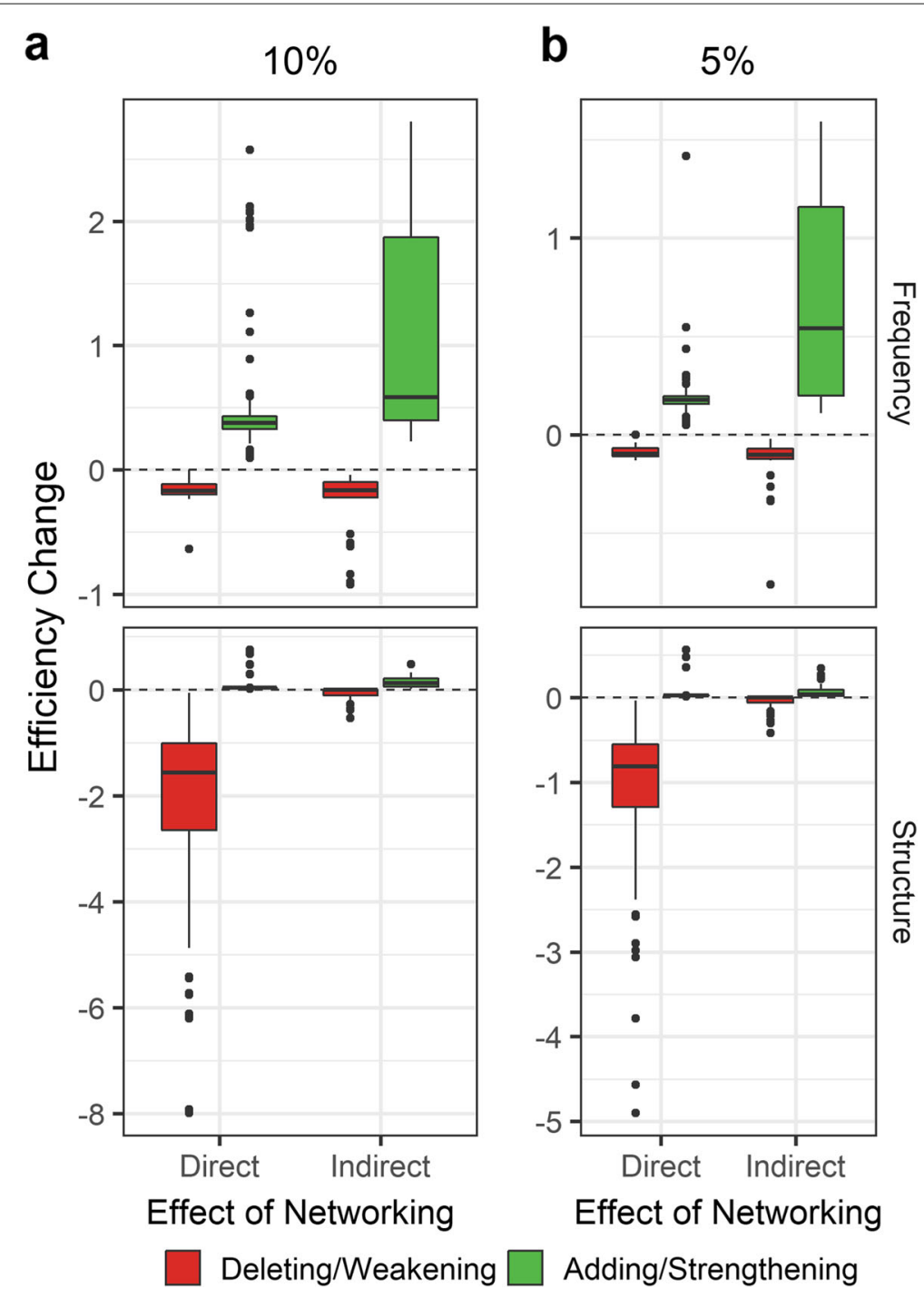

Fig. 8 a Changes in individual's efficiency in case of direct and indirect networking effects based on the difference between the original data and 10\% of deleted/weakened connections (red) as well as $10 \%$ added/strengthened connections (green) in the MANU dataset. b The same quantities in the same dataset based on the difference between the original data and $5 \%$ of deleted/weakened/added/strengthened connections

\section{Abbreviations}

DNC: Email network from the Democratic National Committee; ERGM: Exponential random graph modeling; EU-Dept4: Email network from one of the departments of same European research institute; EU-Inst: Email network from a large European research institute; MANU: Email network from a Polish manufacturer; OSN: Message network from an online social networking site; SAOM: Stochastic actor-oriented modeling

\section{Acknowledgements}

The authors would like to thank Henry K. Dambanemuya, Orsolya Vásárhelyi and Igor Zakhlebin for their helpful comments on an earlier version of the manuscript.

\section{Authors' contributions}

$\mathrm{KT}$ and $\mathrm{AH}$ designed the study. KT performed simulations and analyses. KT and AH wrote the paper. Both authors read and approved the final manuscript.

\section{Funding}

This work was partially funded by the U.S. National Science Foundation under Grant No. IIS-1755873. 
Availability of data and materials

All datasets used in this study are publicly available from KONECT: The Koblenz Network Collection (Kunegis 2013) and SNAP: The Stanford Large Network Dataset Collection (Leskovec and Krevl 2014).

\section{Competing interests}

The authors declare that they have no competing interests.

Received: 14 May 2019 Accepted: 23 August 2019

Published online: 23 September 2019

\section{References}

Akoglu L, Vaz de Melo POS, Faloutsos C (2012) Quantifying reciprocity in large weighted communication networks. In: Proceedings of the 16th Pacific-Asia Conference on Advances in Knowledge Discovery and Data Mining - Volume Part II, PAKDD'12. Springer, Berlin, Heidelberg. pp 85-96

Aral S, Van Alstyne M (2011) The diversity-bandwidth trade-off. Am J Sociol 117(1):90-171

Arrigo F, Benzi M (2016) Updating and downdating techniques for optimizing network communicability. SIAM J Sci Comput 38(1):25-49

Auer P, Cesa-Bianchi N, Fischer P (2002) Finite-time analysis of the multiarmed bandit problem. Mach Learn 47(2):235-256

Barabási A-L (2016) Network Science. Cambridge University Press, Cambridge

Beygelzimer A, Grinstein G, Linsker R, Rish I (2005) Improving network robustness by edge modification. Phys A Stat Mech Appl 357(3):593-612

Block P (2015) Reciprocity, transitivity, and the mysterious three-cycle. Soc Netw 40:163-173

Block P, Stadtfeld C, Snijders TAB (2016) Forms of dependence: Comparing SAOMs and ERGMs from basic principles. Sociol Methods Res 48:1-38

Boccaletti S, Latora V, Moreno Y, Chavez M, Hwang D-U (2006) Complex networks: Structure and dynamics. Phys Rep 424(4-5):175-308

Borgatti SP, Mehra A, Brass DJ, Labianca G (2009) Network analysis in the social sciences. Science 323(5916):892-895

Brandes U (2001) A faster algorithm for betweenness centrality. J Math Sociol 25(2):163-177

Buhl J, Gautrais J, Solé RV, Kuntz P, Valverde S, Deneubourg JL, Theraulaz G (2004) Efficiency and robustness in ant networks of galleries. Eur Phys J B 42(1):123-129

Burt RS (1992) Structural holes: The social structure of competition. Harvard University Press, Cambridge

Burt RS (2002) Bridge decay. Soc Netw 24(4):333-363

Casciaro T, Gino F, Kouchaki M (2014) The contaminating effects of building instrumental ties: How networking can make us feel dirty. Adm Sci Q 59(4):705-735

Chan H, Akoglu L, Tong H (2014) Make it or break it: Manipulating robustness in large networks. In: Proceedings of the 2014 SIAM International Conference on Data Mining. Society for Industrial and Applied Mathematics. pp 325-333. https://doi.org/10.1137/1.9781611973440.37

Chen C, Tong H, Prakash BA, Eliassi-Rad T, Faloutsos M, Faloutsos C (2016) Eigen-optimization on large graphs by edge manipulation. ACM Trans Knowl Discov Data 10(4):49-14930

Dabbish LA, Kraut RE (2006) Email overload at work: An analysis of factors associated with email strain. In: Proceedings of the 2006 20th Anniversary Conference on Computer Supported Cooperative Work. CSCW '06. ACM, New York. pp 431-440

Dijkstra EW (1959) A note on two problems in connexion with graphs. Numer Math 1(1):269-271

Dunbar RIM (1998) The social brain hypothesis. Evol Anthropol Issues News Rev 6(5):178-190

Ebbers JJ (2014) Networking behavior and contracting relationships among entrepreneurs in business incubators. Entrep Theory Pract 38(5):1-23

Engel Y, Kaandorp M, Elfring T (2017) Toward a dynamic process model of entrepreneurial networking under uncertainty. J Bus Ventur 32(1):35-51

Estrada E, Arrigo F (2015) Predicting triadic closure in networks using communicability distance functions. SIAM J Appl Math 75:1725-1744

Estrada E, Hatano N (2008) Communicability in complex networks. Phys Rev E 77:036111

Estrada E, Hatano N, Benzi M (2012) The physics of communicability in complex networks. Phys Rep 514(3):89-119

Faust K (2010) A puzzle concerning triads in social networks: Graph constraints and the triad census. Soc Netw 32(3):221-233

Freeman LC (1977) A set of measures of centrality based on betweenness. Sociometry 40(1):35-41

Garimella K, De Francisci Morales G, Gionis A, Mathioudakis M (2017) Reducing controversy by connecting opposing views. In: Proceedings of the Tenth ACM International Conference on Web Search and Data Mining. WSDM '17. ACM, New York. pp 81-90

Giddens A (1984) The construction of society. University of California Press, Berkeley

Hallen BL, Eisenhardt KM (2012) Catalyzing strategies and efficient tie formation: How entrepreneurial firms obtain investment ties. Acad Manag J 55(1):35-70

He Z-L, Wong P-K (2004) Exploration vs. exploitation: An empirical test of the ambidexterity hypothesis. Organ Sci 15(4):481-494

Jackson TW, Dawson R, Wilson D (2003) Understanding email interaction increases organizational productivity. Commun ACM 46(8):80-84

Kleinbaum AM (2018) Reorganization and tie decay choices. Manag Sci 64(5):2219-2237

Kunegis J (2013) KONECT: The Koblenz Network Collection. In: Proceedings of the 22Nd International Conference on World Wide Web. WWW'13 Companion. ACM, New York. pp 1343-1350

Kuwabara K, Hildebrand CA, Zou X (2018) Lay theories of networking: How laypeople's beliefs about networks affect their attitudes toward and engagement in instrumental networking. Acad Manag Rev 43(1):50-64 
Kwak H, Chun H, Moon S (2011) Fragile online relationship: A first look at unfollow dynamics in twitter. In: Proceedings of the SIGCHI Conference on Human Factors in Computing Systems. CHI '11. ACM, New York. pp 1091-1100

Latora V, Marchiori M (2003) Economic small-world behavior in weighted networks. Eur Phys J B Condens Matter Complex Syst 32:249-263

Latora V, Marchiori M (2001) Efficient behavior of small-world networks. Phys Rev Lett 87(19):198701

Latora V, Marchiori M (2005) Vulnerability and protection of infrastructure networks. Phys Rev E 71:015103

Lazer D, Friedman A (2007) The network structure of exploration and exploitation. Adm Sci Q 52(4):667-694

Leskovec J, Krevl A (2014) SNAP Datasets: Stanford Large Network Dataset Collection. http://snap.stanford.edu/data. Accessed 14 May 2019

March JG (1991) Exploration and exploitation in organizational learning. Organ Sci 2(1):71-87

Mark G, lqbal ST, Czerwinski M, Johns P, Sano A, Lutchyn Y (2016) Email duration, batching and self-interruption: Patterns of email use on productivity and stress. In: Proceedings of the 2016 CHI Conference on Human Factors in Computing Systems. CHI '16. ACM, New York. pp 1717-1728

Mason W, Watts DJ (2012) Collaborative learning in networks. Proc Natl Acad Sci 109(3):764-769

Migliano AB, Page AE, Gómez-Gardeñes J, Salali GD, Viguier S, Dyble M, Thompson J, Chaudhary N, Smith D, Strods J, Mace R, Thomas MG, Latora V, Vinicius L (2017) Characterization of hunter-gatherer networks and implications for cumulative culture. Nat Hum Behav 1:0043

Miritello G, Lara R, Cebrian M, Moro E (2013) Limited communication capacity unveils strategies for human interaction. Sci Rep 3:1950

Mishra K, Boynton L, Mishra A (2014) Driving employee engagement: The expanded role of internal communications. Int J Bus Commun 51(2):183-202

Monge PR, Contractor NS (2003) Theories of communication networks. Oxford University Press, New York

Newman M (2003) The structure and function of complex networks. SIAM Rev 45(2):167-256

Newman MEJ (2001) Scientific collaboration networks. Il. shortest paths, weighted networks, and centrality. Phys Rev E 64:016132

Opsahl T, Panzarasa P (2009) Clustering in weighted networks. Soc Netw 31(2):155-163

Opsahl T, Agneessens F, Skvoretz J (2010) Node centrality in weighted networks: Generalizing degree and shortest paths. Soc Netw 32(3):245-25

Peña J, Brody N (2014) Intentions to hide and unfriend facebook connections based on perceptions of sender attractiveness and status updates. Comput Hum Behav 31:143-150

Raeder T, Lizardo O, Hachen D, Chawla NV (2011) Predictors of short-term decay of cell phone contacts in a large scale communication network. Soc Netw 33(4):245-257

Siciliano MD, Welch EW, Feeney MK (2018) Network exploration and exploitation: Professional network churn and scientific production. Soc Netw 52:167-179

Sommerfeldt EJ, Yang A (2017) Relationship networks as strategic issues management: An issue-stage framework of social movement organization network strategies. Public Relat Rev 43(4):829-839

Squartini T, Picciolo F, Ruzzenenti F, Garlaschelli D (2013) Reciprocity of weighted networks. Sci Rep 3:2729

Tanaka K, Horvát E-A (2019) Networking strategies and efficiency in human communication networks. In: Aiello L, Cherifi H, Lió P, Rocha L, Cherifi C, Lambiotte R (eds). Complex Networks and Their Applications VII - Volume 2 Proceedings The 7th International Conference on Complex Networks and Their Applications COMPLEX NETWORKS 2018. Studies in Computational Intelligence. Springer, Germany. pp 258-270

Tong H, Prakash BA, Eliassi-Rad T, Faloutsos M, Faloutsos C (2012) Gelling, and melting, large graphs by edge manipulation. In: Proceedings of the 21st ACM International Conference on Information and Knowledge Management. CIKM '12. ACM, New York. pp 245-254

Vissa B (2012) Agency in action: Entrepreneurs' networking style and initiation of economic exchange. Organ Sci 23(2):492-510

Waller AD, Ragsdell G (2012) The impact of e-mail on work-life balance. Aslib Proc 64(2):154-177

Watts D, Strogatz S (1998) Collective dynamics of 'small-world' networks. Nature 393(6684):440-442

Watts DJ (2004) Six degrees: The science of a connected age. WWNorton \& Company, Inc., New York

Wolff H-G, Moser K (2009) Effects of networking on career success: a longitudinal study. J Appl Psychol 94(1):196-206

Xu B, Huang Y, Kwak H, Contractor N (2013) Structures of broken ties: Exploring unfollow behavior on twitter. In: Proceedings of the 2013 Conference on Computer Supported Cooperative Work. CSCW '13. ACM, New York. pp 871-876

\section{Publisher's Note}

Springer Nature remains neutral with regard to jurisdictional claims in published maps and institutional affiliations. 